\title{
Diagnosing a rare case of lymphocytic infiltration of the pituitary gland without the invasive procedure of pituitary biopsy
}

\author{
Hamad Haider Khan', Hameed Ullah', Siraj Ulhaq Khan', Huo Guo', Zahid Nadeem³ ${ }^{3}$ Faiz Ullah Khan ${ }^{4}$, \\ Farman Ullah Khan ${ }^{4}$, Shida Hussain ${ }^{5}$, Muhammad Irfan Mansha', Bingyin Shi' \\ ${ }^{1}$ Department of Endocrinology, First affiliated Hospital, Xian Jiaotong University, Xian, China \\ ${ }^{2}$ Department of Radiology, Second affiliated Hospital, Xian Jiaotong University, China \\ ${ }^{3}$ Department of Cardiology, First affiliated Hospital, Xian Jiaotong University, China \\ ${ }^{4}$ Department of Pharmacy Administration and Clinical Pharmacy, School of Pharmacy, Xian Jiaotong University, China \\ ${ }^{5}$ Department of Gastroenterology, Second affiliated Hospital, Xian Jiaotong University, Xian, China
}

\begin{abstract}
Background. Lymphocytic infiltration of the pituitary is an unusual inflammatory disorder of the pituitary and indicated to be autoimmune in origin. Presentations depend on the site of involvement and degree of destruction of the cellular population of the pituitary and may range from an asymptomatic state to pan-hypopituitarism with or without mass effects. In the present study, we represent a rare case of lymphocytic infiltration of the pituitary gland who was diagnosed with such condition and the subsequent management.

Case presentation. A 22-year-old male who was admitted with symptoms of increased thirst and excessive fatigue. Following a thorough physical, clinical, laboratory, biochemistry, and imaging examinations a timely diagnosis of lymphocytic infiltration of the pituitary was made without using the invasive procedure of pituitary biopsy. In light of the diagnosis prompt management with the mainstay of glucocorticoid was started along with replenishing the other deficient hormones. In this study we describe a rare case of lymphocytic infiltration of the pituitary discussing in terms of epidemiology, sign and symptoms, laboratory evaluation, imaging studies, histopathology, management, and the usual outcome. Conclusion. Lymphocytic infiltration of the pituitary gland can be diagnosed with the clinical presentation along with lab evaluation and imaging but without pituitary biopsy, which could help in an early and accurate diagnosis which is the basis for better management of the rare condition.
\end{abstract}

Keywords: lymphocytic infiltration, pan-hypopituitarism, pituitary gland

\author{
Abbreviations \\ MRI - magnetic resonance imaging \\ ALT - alanine transaminase \\ IDBL - indirect bilirubin \\ ALP - alkaline phosphatase \\ DBIL - direct bilirubin \\ AST - aspartate transaminase \\ TBIL - total bilirubin \\ HDL - high density lipoprotein
}

AFP - alpha fetoprotein

hCG - human chorionic gonadotropin

TSH - thyroid stimulating hormone

DI - diabetes insipidus

HRT - hormone replacement therapy

SRS - stereotactic radiosurgery 


\section{INTRODUCTION}

Lymphocytic hypophysitis (LyH) is an uncommon intrasellar lesion characterized by lymphocytic infiltration of the adenohypophysis. In which the immune cells attack the pituitary gland cells to the extent that it causes the gland to under produce or no production of the hormones (1). Evidence suggests that the cause is autoimmune, and the symptoms are usually related to either a mass effect or endocrine dysfunction. The annual incidence of hypophysitis is estimated to be 1 in 7-9 million. Hypophysitis accounts for approximately $0.4 \%$ of pituitary surgery cases (based on a group of large surgical series totaling nearly 10,000 procedures at 5 centers) (2). Lymphocytic infiltration of pituitary mainly has more effect on the female as compared to men, with documented five to one ratio Based on etiology the condition classification can be as primary(idiopathic) or secondary which depends upon presence or absence of other inflammatory conditions related to other systems or advance malignancy treatment with drugs that stimulate inborn immune responses (3). Classification of primary hypophysitis based on histology includes (a) xanthomatous hypophysitis, (b) granulomatous hypophysitis, (c) lymphocytic hypophysitis), (d) necrotizing hypophysitis. Granulomatous - the pituitary gland is infiltration occurs with multiloculated giant cells and histiocytes. Xanthomatous - infiltration of the hypophyseal gland by lipid-rich foam histiocytes, lymphocyte and shows a cystic area of liquefaction. Autoimmune hypophysitis or lymphocytic hypophysitis - The frequent appearance of lymphocytic infiltration of pituitary gland was seen six months postpartum suggesting its correlation with pregnancy however, over the last few years increased number of cases occurring without pregnancy have been documented. Affected patients with this condition usually have the history of other self-immunity disorders in person or a family member (4).

\section{CASE PRESENTATION}

All data were collected anonymously and with the patient's consent. We performed this casethe presentation as per the declaration of the ethics committee of the Xian Jiaotong University, School of Medicine. Oral informed consent was obtained from the patient for publication of this case presentation.

A 22-years aged gentleman who was presented in our hospital complaining of increased thirst and fatigue. Two years ago the patient started having an increased thirst and excessive drinking, up to $10 \mathrm{l} /$ day. Water intake during the day was much more as compared to night with corresponding polyuria.
Apart from that patient experienced increased hair loss including hair loss of the eyebrows, axillary, and pubic area leaving him with a scarce beard. Feeling excessive fatigue, physical decline, and weight loss of $10 \mathrm{~kg}$ in the last 2 years with occasionally experiencing a headache. No nausea, vomiting, anorexia, no polyphagia, hunger, increased bowel movements without fear of cold, memory loss, slow reaction. Owing to all the above-mentioned conditions he did not seek any medical attention, and the following six months, thirst symptoms improved to about 5L of daily water consumption.

Upon routine physical examination the blood pressure was found low, about 80-90/50-60 mm Hg and for further checkup went to a local hospital and MRI of the pituitary in addition to sex hormones assessment was performed and clinical picture pointed out a preliminary diagnosis of Langerhans cell histiocytosis, pituitary adenoma but for better evaluation and subsequent management, he was admitted to our hospital as a case of pituitary adenoma. At the first physical examination, the patient appeared in good general conditions, had no fever, no hypertension, no signs of meningism, and no neurological deficits. Blood pressure was low 90/60mm Hg. Peripheral blood examination was carried out revealed monocyte count of $0.18 \times 10^{9} / 1$ normal range $\left(0.28-0.8 \times 10^{9} / 1\right)$, lymphocyte count of $51.31 \%$ (normal range $20-40 \%$ ), neutrophil count $40.70 \%$ (normal range $50-70 \%$ ), eosinophil 5.70\% (normal range $0-5 \%$ ). Liver function test revealed AST 49.50 (normal range 0-40), ALT 34.20 (normal range 0-40), ALP 69.40 (normal range 36-110), DBIL 2.40 (normal range 0-6), IDBIL 5.0 (normal range 5.1-13.7), TBIL 7.40 (normal range 6-20.5). Serum electrolyte values were as cholesterol 6.42 (normal range 2.4-5.5 $\mathrm{mmol} / \mathrm{l}$ ), uric acid 439.8 (normal range 120-420 $\mathrm{mmol} / \mathrm{l}$ ), Sodium 146.5 (normal range 136-145 $\mathrm{mmol} / \mathrm{l})$, triglyceride 8.60 normal range (0.4-1.6 $\mathrm{mmol} / \mathrm{l}$ ), HDL-c 0.74 (normal range $0.83-1.96 \mathrm{mmol} / \mathrm{l}$ ) and ApoE levels of 84 (normal range $27-45 \mathrm{mg} / \mathrm{l}$ ). AFP of 1.39 (normal range $0.00-7.02 \mathrm{ng} / \mathrm{ml}$ ) and hCG of less than $0.50 \mathrm{mIU} / \mathrm{l}$. Thyroid function test values were FT4 12.2 (normal range 9.05-25.0 pmol/l), FT3 7.38 normal range 2.91-9.08 pmol/l) and TSH 1.59 (normal range 0.25-5 uIU/l), morning cortisol levels of 3.49 normal range 5.0-28.0 ug/dl) (Table 1). The first pituitary function works up showed a low hormonal level (figure 1).

Multi-phase dynamic contrast-enhanced MRI of the pituitary scan was performed showing abnormal enhancement of pituitary stalk and gray nodule indicating enlarged pituitary stalk. Pituitary MRI showing homogenous enlarged pituitary stalk and posterior pituitary significantly reduce. Differential diagnosis was carried out with tests that ruled out other causes and specific hormonal, baseline tests 
Table 1. Peripheral blood examination

\begin{tabular}{|l|c|c|}
\hline Variable & Values & Reference range \\
\hline WBC & 5.61 & $4-10 \times 10^{9} / \mathrm{I}$ \\
\hline Lymphocyte percent & 51.31 & $20-40 \%$ \\
\hline Lymphocyte count & 2.88 & $1.5-4 \times 10^{9} / \mathrm{I}$ \\
\hline Eosinophil percent & 5.70 & $0.0-5 \%$ \\
\hline Eosinophil count & 0.32 & $0.0-0.45 \times 10^{9} / \mathrm{I}$ \\
\hline Monocyte percent & 2.10 & $3-8 \%$ \\
\hline Monocyte count & 0.12 & $0.2-0.8 \times 10^{9} / \mathrm{I}$ \\
\hline Neutrophil percent & 40.70 & $50-70 \%$ \\
\hline Neutrophil count & 2.28 & $2-7 \times 10^{9} / \mathrm{I}$ \\
\hline Basophil percent & 0.20 & $0-1 \%$ \\
\hline Basophil count & 0.01 & $0-0.2 \times 10^{9} / \mathrm{I}$ \\
\hline Uric acid & 439.80 & $120-420 \mathrm{umol} / \mathrm{I}$ \\
\hline Sodium (Na) & 146.50 & $136-145 \mathrm{mmol} / \mathrm{I}$ \\
\hline Triglycerides & 8.60 & $0.4-1.6 \mathrm{mmol} / \mathrm{l}$ \\
\hline HDL & $0 . .74$ & $0.83-1.96 \mathrm{mmol} / \mathrm{I}$ \\
\hline AST & 49.50 & $0-40 \mathrm{u} / \mathrm{l}$ \\
\hline ALT & 34.20 & $0-40 \mathrm{u} / \mathrm{l}$ \\
\hline ALP & 69.40 & $36-110 \mathrm{u} / \mathrm{l}$ \\
\hline DBIL & 2.40 & $0-6 \mathrm{umol} / \mathrm{l}$ \\
\hline IDBIL & 5.00 & $5.1-13.7 \mathrm{umol} / \mathrm{I}$ \\
\hline TBIL & 7.40 & $6-20.5 \mathrm{umol} / \mathrm{I}$ \\
\hline AFP & 1.39 & $0.0-7.02 \mathrm{ng} / \mathrm{ml}$ \\
\hline hCG & $<0.50$ & $\mathrm{mlU} / \mathrm{ml}$ \\
\hline
\end{tabular}

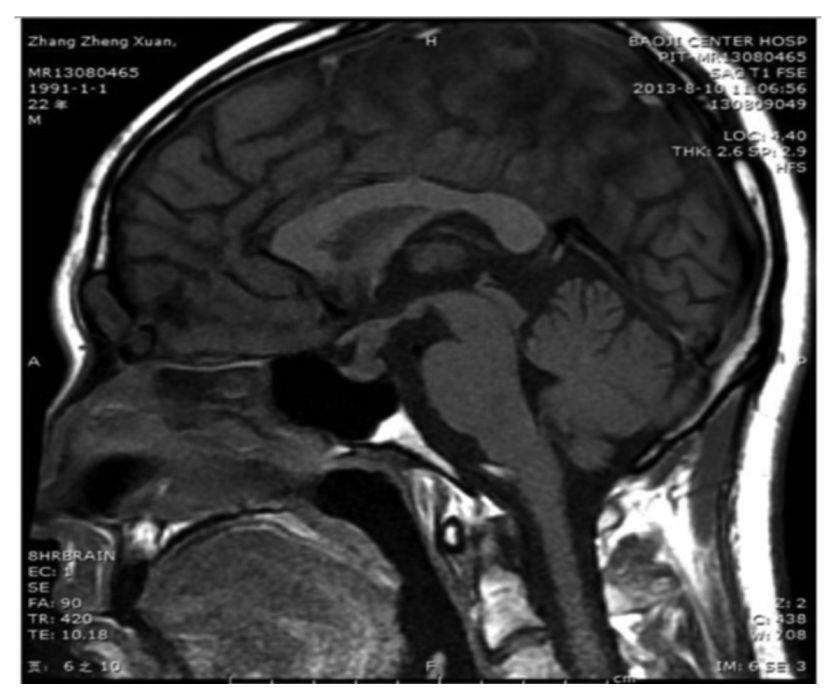

FIGURE 1. Initial sagittal T1 brain MRI 1

were carried out which could be essential tools in diagnosing lymphocytic hypophysitis without the need for any invasive procedure like a pituitary biopsy.

\section{DISCUSSION}

In this case report study, we put forward presentation of a very challenging case, a male patient of 22 years age who was affected by lymphocytic infiltration of the pituitary who presented with homogenous enlargement of the hypophyseal stalk on contrast-enhanced MRI, this case we presented is noteworthy because it helps us elucidate certain aspects of the natural history of lymphocytic infiltration of the pituitary, diagnostic measures used in early and prompt diagnosis, and the issue of glucocorticoid therapy. The most common type of hypophysitis being lymphocytic hypophysitis. Classification of lymphocytic infiltration of the pituitary can be divided based on etiology and also on anatomical grounds. Etiologically lymphocytic hypophysitis is classified into two types: primary and secondary. Primary if no specific associated etiologic factors are found and secondary is defined by the presence of systemic disease or with local lesions of the sella (5). Based on the anatomy of the pituitary the lymphocytic hypophysitis with the presence of lymphocytic infiltration, lymphocytic hypophysitis can be divided into lymphocytic adenohypophysitis if the anterior pituitary is infiltrated, lymphocytic neurohypophysitis given that the posterior pituitary is involved, lymphocytic infundibulohypophysitis if the pituitary stalk is affected or lymphocytic panhypophysitis if the whole of pituitary if found to have significant findings suggesting the lymphocytic infiltration of the pituitary (6). Lymphocytic infiltration of the pituitary, considered as uncommon autoimmunity condition of the hypophysis and first reporting of the condition is dated to be fifty years ago (7). Mostly the condition is known to affect young females, mostly pregnant but it can be presented in males and females irrespective of age (811). The condition usually presents as a mass in the sella which causes signs/symptoms of (headache, impairment of visuals, paralysis of the eyeball muscles) as well as decreased functioning of the hypophysis (panhypopituitarism, diabetes insipidus, hyperprolactinemia) $(3,12)$. Diagnosis of lymphocytic hypophysitis can be challenging as it may mimic many other relatable conditions so confirm diagnosis can be made based on the presenting sign and symptoms in conjunction with biochemical evaluation, hormonal analysis, imaging and in some cases in pituitary biopsy if the diagnosis is still in question based on the other non-invasive measures. On imaging, findings indicate pituitary enlargement with symmetrical expansion and homogenously enhanced hypophysis. Hypophyseal stalk seen thickened but without deviation. A pituitary biopsy is an invasive technique and brought into consideration if the non-invasive measures fail to give us strong evidence of a specific diagnosis. The pituitary biopsy is the definite way of making specific diagnosis upon apparent lymphocytic infiltration of the pituitary. As its invasiveness is brought into consideration most of the time the pituitary biopsy is not performed in patients rendering the cases remain unclear and for that purpose serological biomarker would be very helpful in this challenging condition. 
In our case on contrast-enhanced MRI homogenous enhancement of the pituitary was found along the thickened hypophyseal stalk (figure 2). Our patient had symptoms of occasional headache and on the hormonal analysis found to be having decreased function of the pituitary. The natural history of lymphocytic infiltration of the pituitary cannot be predicted however mostly benign and resolution occurring unexpectedly in a few cases presented. Nearly all presenting patient require high-dose glucocorticoid(pulse dose followed by gradual tapering) while patients with pituitary dysfunction accompanying DI requires HRT (13). Of the patients who do not respond to medications or those suffering from mass effects or of enlarged pituitary are candidates of the trans-sphenoidal resection of the sellar mass causing neurological symptoms. Surgical resection has the additional advantage of pro-

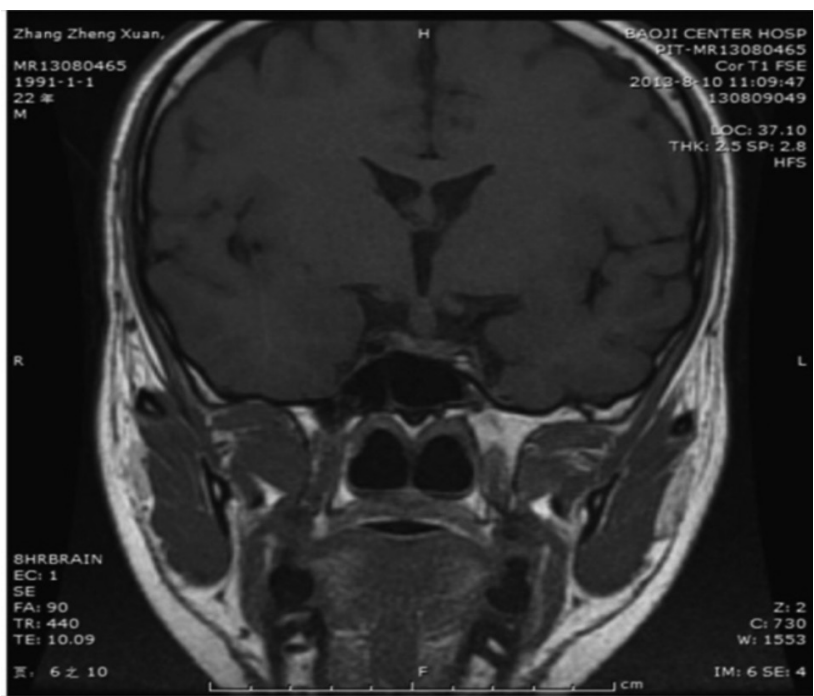

FIGURE 2. Initial coronal T1 brain MRI 2

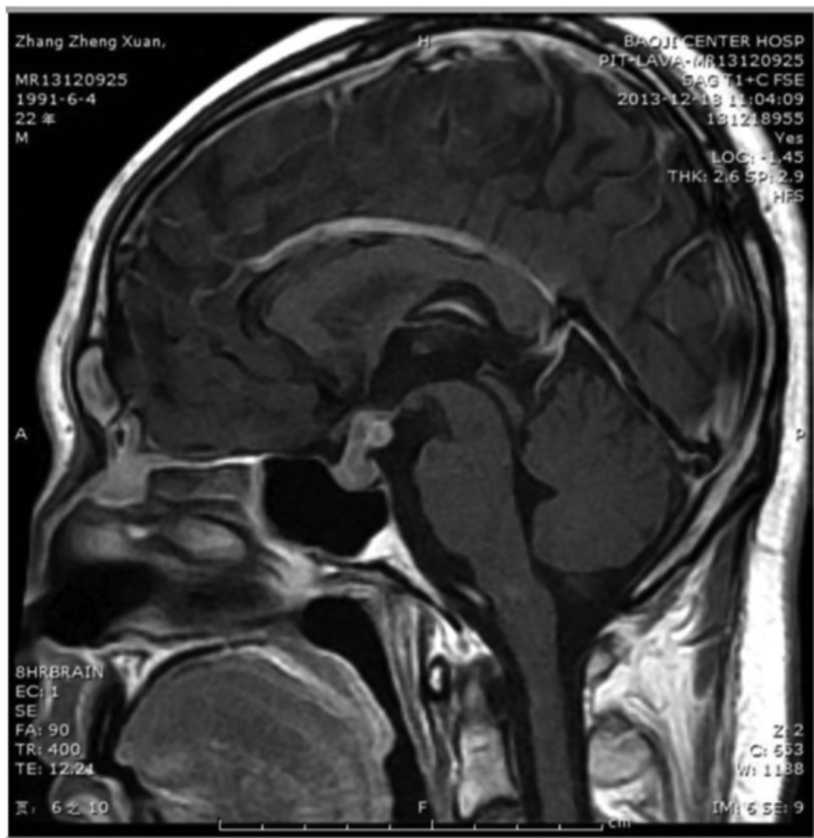

FIGURE 3. Initial sagittal T1 brain MRI 3 viding biopsy for histological identification as well as good relief from headaches with signs and symptoms of vision problems $(14,15)$. In our patient because of the decreased pituitary function and importance of glucocorticoid therapy prednisolone along with sex hormone was prescribed in the prospect of reducing the enlargement of the pituitary stalk. Medications other than glucocorticoid include other modalities such as therapy of immunosuppression consisting of methotrexate(MTX) cyclosporine, rituximab, or azathioprine. The prescription of our patient was as (1) prednisone $15 \mathrm{mg} 3$ times a day (2) antler $40 \mathrm{mg} 2$ times a day, (3) ranitidine 0.152 times a day, (4) calcium carbonate 600 mg 1 time daily, (5) inosine tablets 0.43 times a day, (6) fenofibrate $200 \mathrm{mg} 1$ time a day. Intially the manifestations resolved within the first three weeks of diagnosis and recommended therapy, but lately recurrent with moderate symptoms and afterall retrieved by radiotherapy.

Cases failed to respond to conventional therapy are then referred to SRS radiotherapy. SRS is a sort of radiation therapy allowing more accurate localization with precise dosage deliverance with a lesser sum consisting of customary tissue effect as compared to conventional therapy. Stereotactic radiosurgery use is common in cases presenting with sella turcica tumors or abnormalities of the vessels. SRS characteristically provides means for effectual management alternative in controlling the tumor and somewhat normalizes in the cases presenting with hyperfunctioning pituitary adenoma and the most common complication reported is hypopituitarism later in the treatment (16-18). Poor prognosis of lymphocytic infiltration of the pituitary is mainly due to or can be associated with poor pituitary function, of which adrenal insufficiency is most probable and most common cause of death. In early stage early detection and management can protect the pituitary function and while in chronic when hypopituitarism of varying degree occurs, administration of long term or lifelong hormonal replacement is management principle to avoid severe deficit physiologic function of endocrine gland.

\section{CONCLUSIONS}

We reported an rare case of the lymphocytic Infiltration of the pituitary gland whose diagnosis was made based on the clinical presentation along with lab evaluation and imaging but without the need for an invasive procedure like a pituitary biopsy. The highlighting of this case report is that patients presenting with pituitary disorders, who present with enlargement of the hypophysis on imaging and clinical manifestation of decreased functioning of pituitary no matter if isolated or whole, importantly 
the consideration of possible lymphocytic infiltration of the pituitary should be bear in mind. It is also emphasized that an early and thorough examination leads to early and accurate diagnosis which is the basis for better management of the rare condition.

\section{REFERENCES}

1. Quencer RM. Lymphocytic adenohypophysitis: autoimmune disorder of the pituitary gland. American Journal of Neuroradiology. 1980;1(4):343-5.

2. Faje A. Hypophysitis: Evaluation and Management. Clin Diabetes Endocrinol. 2016 Sep 6;2:15.

3. Glezer A, Bronstein MD. Pituitary autoimmune disease: nuances in clinical presentation. Endocrine. 2012;42(1):74-9.

4. Beressi N, Cohen R, Beressi JP, Dumas JL, Legrand M, Iba-Zizen MT, et al. Pseudotumoral lymphocytic hypophysitis successfully treated by corticosteroid alone: first case report. Neurosurgery. 1994;35(3):5058; discussion 8.

5. Ozbey N, Sencer A, Tanyolac S, Kurt R, Sencer S, Bilgic B, et al. An intrasellar germinoma with normal cerebrospinal fluid beta-HCG concentrations misdiagnosed as hypophysitis. Hormones. 2006;5(1):67.

6. Annamaria DB, Fahrettin K, Antonio Agostino S, Giuseppe R, Gilda T, Marina B, et al. Anti-hypothalamus and anti-pituitary antibodies may contribute to perpetuate the hypopituitarism in patients with Sheehan's syndrome. European Journal of Endocrinology. 2008;158(2):147-52.

7. Goudie RB, Pinkerton PH. Anterior hypophysitis and hashimoto's disease in a young woman. The Journal of Pathology and Bacteriology. 1962;83(2):584-5.

8. Takahashi Y. Autoimmune hypophysitis: new developments. Handbook of Clinical Neurology. 2014;124(124C):417.

9. Fukuoka H. Hypophysitis. Endocrinology \& Metabolism Clinics of North America. 2015;44(1):143-9.

10. Kartal I, Yarman S, Tanakol R, Bilgic B. Lymphocytic panhypophysitis in a young man with involvement of the cavernous sinus and clivus. Pituitary. 2007;10(1):75.

\section{Acknowledgment}

We (authors) are thankful to the hospital administration.

Conflict of interest: none declared Financial support: none declared

11. Gellner V, Kurschel S, Scarpatetti M, Mokry M. Lymphocytic hypophysitis in the pediatric population. Childs Nerv Syst. 2008;24(7):785-92.

12. Falorni A, Minarelli V, Bartoloni E, Alunno A, Gerli R. Diagnosis and classification of autoimmune hypophysitis. Autoimmunity Reviews. 2014;13(4-5):412-6.

13. Yamagami K, Yoshioka K, Sakai H, Fukumoto M, Yamakita T, Hosoi M, et al. Treatment of lymphocytic hypophysitis by high-dose methylprednisolone pulse therapy. Internal Medicine. 2003; 42(2):168-73.

14. Leung GK, Lopes MB, Thorner MO, Vance ML, Jr LE. Primary hypophysitis: a single-center experience in 16 cases. Journal of Neurosurgery. 2004;101(2):262-71.

15. Laws ER, Vance ML, Jr JAJ. Hypophysitis. Pituitary. 2006;9(4):331-3.

16. Caputo C, Bazargan A, Mckelvie PA, Sutherland T, Su CS, Inder WJ. Hypophysitis due to IgG4-related disease responding to treatment with azathioprine: an alternative to corticosteroid therapy. Pituitary. 2014;17(3):251-6.

17. Bellis AD, Pane E, Bellastella G, Sinisi AA, Colella C, Giordano R, et al. Detection of antipituitary and antihypothalamus antibodies to investigate the role of pituitary or hypothalamic autoimmunity in patients with selective idiopathic hypopituitarism. Clinical Endocrinology. 2011;75(3):361-6.

18. Minniti G, Osti MF, Niyazi M. Target delineation and optimal radiosurgical dose for pituitary tumors. Radiation Oncology. 2016;11(1):135. 